\title{
Balance Disorder Rehabilitation through Movement Interaction
}

\author{
Juan Enrique Garrido, Irma Marset \\ Computer Science Research Institute \\ University of Castilla-La Mancha \\ Albacete, Spain \\ juanenrique.garrido@uclm.es; irma.marset@alu.uclm.es
}

\author{
Víctor M.R. Penichet, María D. Lozano \\ Computer Systems Department \\ University of Castilla-La Mancha \\ Albacete, Spain \\ \{victor.penichet, maria.lozano\}uclm.es
}

\begin{abstract}
Neuro-rehabilitation process usually implies the patients' need of going to medical centers. This situation makes patients go out of their daily life environment and routine. In this way, authors have developed a system that allows balance disorder patients to perform a specific rehabilitation exercise (to control the balance when walking) at home. Thanks to that, the patients are able to recover from their disability in a more comfortable and appropriate environment always under the physiotherapist supervision. In this sense, the system complements the physiotherapists' work, avoiding in many cases the need of their presence during the repetition of the rehabilitation exercise. To that end, the system is based on movement interaction through Kinect devices and a specific framework which analyzes the patients' movements in order to generate adequate indications and corrections during the rehabilitation process. Medical staff finds the system as an assistant tool with which enables to control the rehabilitation process in the distance if patients' conditions allow.
\end{abstract}

Healthcare; rehabilitation; Kinect; movement interaction; hci

\section{INTRODUCTION}

Nowadays, more and more doctors, physiotherapists and healthcare centers incorporate in their rehabilitation work therapies, videogames and, more specifically, the technologies and methods used in them [1][2][3]. The result is a process which allows patients to recover affected functionalities through an entertaining, useful and effective way. Neurorehabilitation [4] is one of the areas in which this new type of therapies has been used in the last few years. In particular, this new rehabilitation procedure has been applied to people with brain damage caused by cardiovascular accident, brain attack and neurodegenerative diseases such as Parkinson, multiple sclerosis and Alzheimer. This therapy, even though being currently unconventional, has demonstrated remarkable benefits in the applied rehabilitations [1][2]. Specifically, a project conducted with multiple sclerosis patients at the Rey Juan Carlos University [5] revealed as results a remarkable improvement when using exercises based on videogames. Thanks to the new therapy, many patients have been able to return completely to daily life activities which were previously very difficult for them, such as walking, jumping or standing up without overbalancing.

The use of movement interaction is one of the most popular elements of videogames and it has to be underlined as a new foundation to be applied in healthcare. Concretely, this interaction allows the creation of systems that offer patients in need the possibility of completing their rehabilitation process at home by following the steps which have been indicated by qualified medical staff.

The authors have developed a system which takes into account how movement interaction can improve the rehabilitation process. Concretely, the system focuses on the rehabilitation of patients with neurodegenerative diseases or some brain damage (e.g. traumatic brain injury, dementia, cerebral palsy, etc.). This type of patients is able to do many of these exercises, such as to get up or sit in a chair, to walk straight, to pick up or move objects, etc. Specifically, the system is centered on helping patients to rehabilitate balance disorder. These simple actions for people without any disease are complex and imply a costly learning process for people with neurodegenerative and brain diseases. Therefore, the rehabilitation and training in these cases represents an essential and important improvement milestone.

The capacity of detecting movement enables to track the patients' movement in order to analyze them. The analysis result provides enough information to perform corrective actions, which include data collecting. The correction can be obtained by comparing how each rehabilitation exercise is really done with regard to the perfect way to perform it. Accordingly, if the result is correct then the system communicates it to the patient; otherwise the system has to create specific advises and clarifications in the form of animations in order to correct the way to complete the rehabilitation process. Regarding the data collection, the information about patients' evolution in each exercise allows to create a useful statistic study for the medical staff. The aim of this additional functionality is to be able to know the current state of each patient's rehabilitation process.

Kinect ${ }^{1}$ is the device used in the developed system to work with movement interaction. The device implies important advances due to the fact that users have the capacity to interact with systems through natural and common gestures. The medical world has found Kinect to be an adequate tool to experiment with in order to reach solutions and get improvements. A wide range of aspects have been treated, from haptic problems [6] to the rehabilitation of chronic diseases [7]. The system is generalizable to similar movement

\footnotetext{
${ }^{1}$ Kinect for Windows SDK, 2012: http://www.microsoft.com/enus/kinectforwindows/
} 
interaction devices such as Asus Xtion Pro Live ${ }^{2}$ y SoftKinect DepthSense ${ }^{3}$.

The paper is organized as follows: Section 2 describes the developed system for rehabilitation at home, its functionality and interface. And Section 3 presents conclusions and future work.

\section{BALANCE DISORDER REHABILITATION SYSTEM}

Considering how new technologies, and more specifically, new interactions modes can improve the conditions of patients in rehabilitation processes, authors have developed a system focused on a specific exercise for people affected by neurodegenerative problems. The exercise consists in helping patients to recover from a balance disorder by training them to walk straight, which shows how the system is focused on a very specific objective. They are able to complete their therapy at home, performing each exercise defined by the medical staff, without the need of taking a trip to specific centers. The main consequence is the achievement of incorporating the therapy in the daily life environment of each patient. Over time, the system will be part of the patients' home becoming a common element and the rehabilitation another daily activity.

According to the information obtained in interviews and meetings with specialists of the physiotherapy field, one of the keys to the success in the recovery of the patient with balance disorder, along with many other common problems, relies on the continuous repetition of the related rehabilitation exercise. It implies a weighty task, and a great challenge for the patient. These exercises are apparently simple but entail a great complexity for the patients. In most cases the problem stems from the fact that the patients are not aware that they are losing the balance in one side of their body, if they are straight, etc. The proposed system monitors the patient in real time and $\mathrm{s} /$ he can see him/herself in a projection which helps constantly to perform the exercise series defined by the medical staff.

The system presents a well-defined deployment in order to be able to offer the new rehabilitation conditions. In particular, the system deployment is based on two connected points: patient's home and the server. The first one represents the place where each Kinect device operates. In the patients' home the systems contains a personal computer connected to a Kinect device and to a screen. The personal computer has to be connected to Internet in order to be able to establish connection with the server. The screen shows the system interface, the system part responsible for guiding the patients during the rehabilitation process. For its part, the server offers and receives information from the software that operates in the personal computer. Specifically, the received information is the statistics for each performed exercise and the offered information is the parameters to customize the exercise for the current patient (defined by the medical staff).

2 ASUS Xtion Pro Live, ASUS, 2012:

http://www.asus.com/Multimedia/Motion_Sensor/Xtion_PRO_LIVE/

${ }^{3}$ SoftKinetic DepthSense, SoftKinectic, 2012:

http://www.softkinetic.com/Solutions/iisuSDK.aspx
The proposal presents remarkable benefits from two points of view. Firstly, from the medical staff perspective, who find the system an adequate tool with which to gain time. Such overtime increases the medical staff capacity to attend patients more intensively. The system partially replaces them during the exercise performance, and then they are able to be centered on the results and the way to improve each rehabilitation process. The related rehabilitation exercise usually represents many repetitions. This fact implies that the constant presence of the physiotherapist is not required due to the indications of the system. Those indications offer enough information to the patient to know what he is performing wrong and how to correct the action. In this way, the system contains different ways to alert the patient without overflowing him. The easiest indications and generally the most common ones appear at the screen giving color to the exercise part which is being badly completed. For example, the system shows with an arrow the way to correct the balance (see Fig. 1-D). Other indications will be more intrusive if the problem to correct can affect the patient's health. For example the patient can fall if he does not correct the current action. Therefore, taking into account that the management of the physiotherapist is essential, thanks to the system the patient can repeat the exercise and implied movements again and again without the need for the physiotherapist to be dedicated full time. Even so, it is possible to think that the patients lose some personalization level without the presence of the medical staff, which is in many cases desirable, but, instead, the patients are able to progress at home with their family or people close to them.

The patient composes the second point of view. The system avoids trips or movements to medical or specific centers where the rehabilitation process has to be performed. In this way, the patients can be at home in a well-known and more comfortable environment; something that would certainly contribute their physiological benefit. Additionally, the patient can perform the rehabilitation process in an independent and less invasive way without the need to depend on third parts more than the strictly necessary. Therefore, they can organize their own progress at leisure based on steps marked by their medical staff. This benefit and the previous one suppose a significant progress in the rehabilitation aspect, if we take into account the fact of there are patients who have to perform the rehabilitation process during the rest of their life.

Following, the next subsections describe two essential elements of the system: (1) the system functionality indicating the development foundations and how it works; and (2) the system interface which is needed in order to offer a system that helps the patients and the physiotherapists in the best possible way.

\section{A. System Functionality}

The objective of the system is to achieve checking without interruption the posture of the patient during the exercise. The postures identification is made by the use of the development kit that Microsoft provides [8]. This process is a key element, so explaining how the system performs the identification is essential. The SKD allows the recognition of all the body joints that Kinect is able to identify, concretely 20. These 
points are provided to the system through a class that represents the skeleton and offers an accessible way to them. Once the system obtains this class, the next step is to search for those points which are necessary to identify correct postures, by collecting their position in the obtained parameters. Each position is stored in a specific class composing a set of three values referring the position of the point in the space ( $\mathrm{x}, \mathrm{y}$ and $\mathrm{z}$ axes). Once the positions have obtained this representation, the detection of the patient' posture starts while he is walking. To this end, the system considers that there are pairs of body parts that may be aligned with a little error rate. These pairs are those which are at the same height in the patients' body. For example, the points related to the shoulders have to be at the same height during the exercise; otherwise, the patient is inclined to the side related to the shoulder which is in a lower position. The overall described functionality is gathered under a specific framework which performs the analysis based on relative points what makes it independent of the patients' height.

The patient has to perform the exercise in a three meterlong space. He has to go from one initial point to the final one following a straight virtual line. The system shows the patient himself through a screen during the exercise, with information about how the exercise is being performed. Additionally, the screen indicates corrections and advices if some error is detected during the procedure. The next subsection describes completely what information and signals are shown in order to help patients in the completion of the exercise. These information and advices come as a result of the described analysis that the system continuously makes for each patient's posture when walking straight.

\section{B. System Interface}

The interface is an essential element of the system due to the importance in patients' acceptance. They need to find a rehabilitation system which guides them adequately during the exercise under the physiotherapist's supervision. As a result, the system will be able to be incorporated in their daily life as a natural element of their own home. In this sense, the system can guide each patient step by step, as the physiotherapists would do. Additionally, the interface can incorporate enough information to complete the specific exercise and, also, to help, guide and correct users at any point of the process.

The system interface is based on the interaction provided by Kinect. The interface follows the generic usability guidelines for any application and the ones that are defined by the used device as well. The first ones refer to the visual aspect of the system so that its appearance avoids uncomfortable work ways. And the Kinect guidelines point the way to create helpful interfaces controlled by natural movements such as hand and head movements.

The user type is a key condition when analyzing the needs and appearance of the interface. The patients can be people who know perfectly the current technology or people who know nothing about it. In this sense, the analysis has indicated

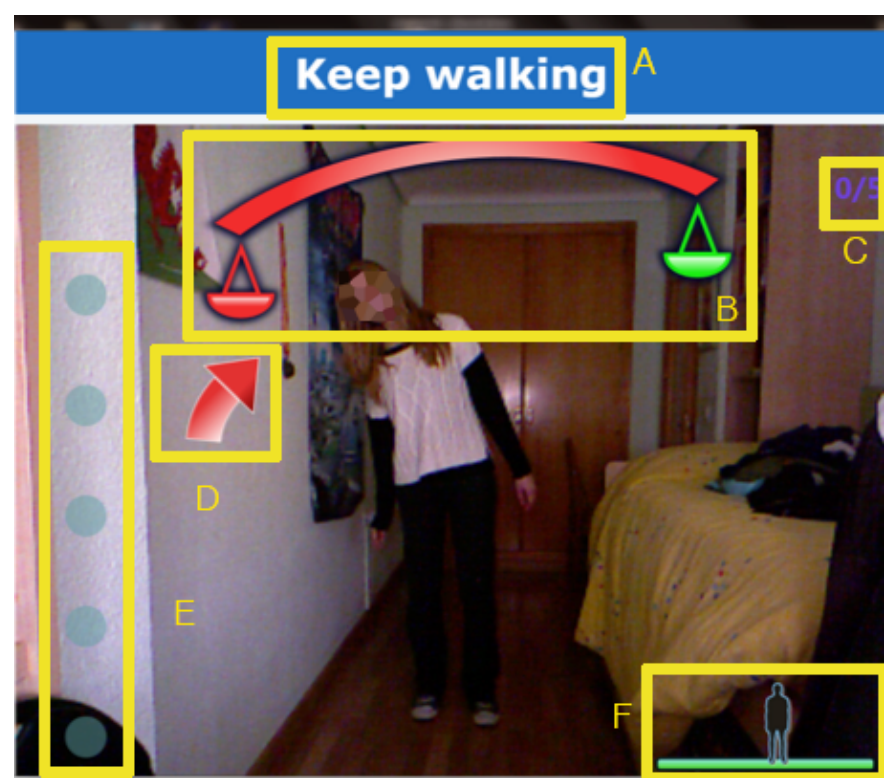

Figure 1. System screenshot which shows user instructions and advices in order to complete the rehabilitation exercise

that the interface must show as foundations a well-defined set of advices, notifications and signals. At the same time, the system can show the information on the screen together with adequate metaphors in order to complete the indications. Therefore, the system has to allow patients to know every time what to do exactly and how, particularly since the exercise evolution is controlled by the patients' interaction.

Fig. 1 shows a screenshot of the system in which it is possible to note the offered information to the patients during the rehabilitation process. Following, each element of the interface is explained:

- Current task of the exercise to be performed (Fig. 1A): the patients need to know what to do at any time. The system analyzes the evolution of the exercise by studying the distance covered by the patient. Firstly, the system checks that the user is at the starting point. While the patient is not at the needed point, the system indicates that $\mathrm{s} / \mathrm{he}$ has to be at that point. Following, the system indicates that the next step is to walk until the end of the rehabilitation route. Finally, the system determines the end of the exercise when the patient reaches the end of the virtual line that has been followed.

- Balance level (Fig. 1-B): this mark is a key piece of information. A common balance that inclines to the side on which the patient is inclining the body while walking shows the equilibrium. This is an effective manner to show the correctness of the position. If the user balances excessively the body, the balance is inclined and the color of the affected side changes to red, as it is shown in Fig. 1.

- Attempt and its percentage (Fig. 1-C and 1-E): the system maintains the attempts the user has to complete (based on the physiotherapist's indications) and shows the number and percentage of complete ones. 


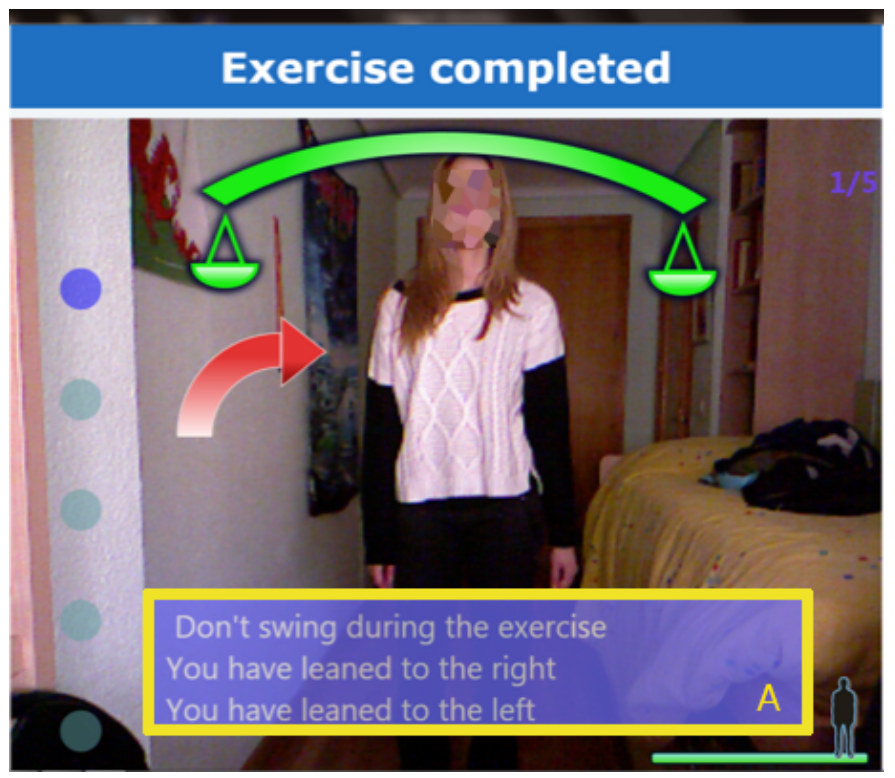

Figure 2. System screenshot which shows an overview of a complete rehabilitation exercise

- Corrective arrows (Fig. 1-D): indicating the side to which the patient has to correct the posture is a simple way to help the user during the exercise. Fig. 1 shows how the patient must correct his postures to the adequate side.

- Completion degree of the whole exercise (see Fig. 1F): the patient has to know at what point of the exercise he is. This signal marks a man in a line showing the evolution. The left is the starting point and the right the final one.

- Conclusions (Fig. 2-A): this information appears when the patients finished each attempt. This element gives a list of the things to correct in the next step. If the attempt is performed correctly, the system congratulates the patient.

Finally, incorporating the possibility to control some functionality through the user voice has completed the interface. This capacity is thanks to the Kinect device, which can interact with users using their voice by offering a microphone component and related software with recognition capability. This fact creates a better environment to users with arm movement problems, in which they are able to manage the interface with voice indications. Primarily, it allows the user to manage the system with preferences or simple actions such as indicating that he is prepared to start.

\section{CONCLUSIONS AND FUTURE WORK}

In this paper, we have presented a system to improve the rehabilitation conditions of people with balance disorder. In particular, the system serves as an assistant for patients focusing on a specific rehabilitation exercise: to train in walking straight. The main contribution comes from the fact that it allows the patients to perform the rehabilitation process at home under the supervision of physiotherapists. They are able to incorporate the system into their daily life environment and rehabilitate their disabilities in a more comfortable setting. As a result, patients avoid trips and displacements to medical centers. Additionally, medical staff obtains an assistant which makes his presence not necessary in many cases when there is a need to repeat constantly the exercise. The physiotherapists can define specific parameters of the exercise for each patient, control his evolution and study in depth the obtained results.

The proposal is based on movement interaction through Kinect devices and a framework which analyses the patients' movements. The framework studies how each patient performs the exercise and depending on it creates adequate indications to correct and guide him. These indications are fundamental in order to complete the supervision of medical staff. In this way, the system interface turns into an essential component due to the necessity to get the patients' acceptance. Therefore, authors have emphasized the way the system interacts together with a correct analysis of how patients perform the exercise.

The system presents interesting future work. Currently, authors are working on getting a more friendly and intuitive system for the patients. The idea is to offer an interaction as natural as possible with the system and then, to obtain a greater level of acceptance. Additionally, further efforts are being made in order to obtain a better deployment of the system. The objective is to offer to the user an application easy to install at home.

\section{ACKNOWLEDGMENT}

We would like to acknowledge the project CICYT TIN2011-27767-C02-01 from the Spanish Ministerio de Economia y Competitividad and the Regional Government Junta de Comunidades de Castilla-La Mancha PPII10-03004174 and PII2C09-0185-1030 projects for partially funding this work.

\section{REFERENCES}

[1] Parry, I.S., Bagley, A., Kawada, J., Greenhalgh, D.G., Palmieri, T.L.: Commercially available interactive video games in burn rehabilitation: therapeutic potential, Burns, Vol. 38 (4), pp. 493-500, June 2012.

[2] Smith, S.T., Schoene, D.: The use of exercise-based videogames for training and rehabilitation of physical function in older adults: current practice and guidelines for future research, Aging Health, Vol. 8, pp. 243-252, June 2012.

[3] Marston, H.R., Smith, S.T.: Interactive Videogame Technologies to Support Independence in the Elderly: A Narrative Review, Games for Health Journal, Vol. 1(2), pp. 139-152, April 2012.

[4] van Paasschen, J., et al.: Cognitive Rehabilitation Changes MemoryRelated Brain Activity in People With Alzheimer Disease, Neurorehabil Neural Repair January 2013.

[5] Luna-Oliva, L., et al.: Evaluation of the Use of a Virtual Reality VideoGame System as a Supplement for Rehabilitation of Children with Cerebral Palsy, Biosystems \& Biorobotics, Vol. 1, pp 873-877, 2013.

[6] Shrewsbury, B.: "Providing Haptic Feedback Using the Kinect", In Proc. ASSETS'11, Dundee, Scotland, 2011, 321-322.

[7] Schonauer, C.: Chronic pain rehabilitation with a serious game using multimodal input, 2011 International Conference on Virtual Rehabilitation (ICVR), 1-8.

[8] Shotton, J., et al.: Real-Time Human Pose Recognition in Parts form Single Depth Images, 2011 IEEE Conference Computer Vision and Pattern Recognition (CVPR), 1297-1304. 